\title{
Clinical Efficacy of Electroneurography in Acute Facial Paralysis
}

\author{
Dong-Hee Lee \\ Department of Otolaryngology-Head and Neck Surgery, Uijeongbu St. Mary's Hospital, The Catholic University of Korea, Uijeongbu, Korea
}

Received September 23, 2015

Revised December 5, 2015

Accepted February 13, 2016

\author{
Address for correspondence \\ Dong-Hee Lee, MD \\ Department of Otolaryngology- \\ Head and Neck Surgery, \\ Uijeongbu St. Mary's Hospital, \\ The Catholic University of Korea, \\ 271 Cheonbo-ro, Uijeongbu 11765 , \\ Korea \\ Tel +82-31-820-3820 \\ Fax +82-31-847-0038 \\ E-mail1eedh0814@catholic.ac.kr
}

The estimated incidence of acute facial paralysis is approximately 30 patients per 100000 populations annually. Facial paralysis is an extremely frightening situation and gives extreme stress to patients because obvious disfiguring face may cause significant functional, aesthetic, and psychological disturbances. For stressful patients with acute facial paralysis, it is very important for clinicians to answer the questions like whether or not their facial function will return to normal, how much of their facial function will be recovered, and how long this is going to take. It is also important for clinicians to treat the psychological aspects by adequately explaining the prognosis, in addition to providing the appropriate medical treatment. For decades, clinicians have used various electrophysiologic tests, including the nerve excitability test, the maximal stimulation test, electroneurography, and electromyography. In particular, electroneurography is the only objective measure that is useful in early stage of acute facial paralysis. In this review article, we first discuss the pathophysiology of injured peripheral nerve. And then, we describe about various electrophysiologic tests and discuss the electroneurography extensively.

J Audiol Otol 2016;20(1):8-12

KEY WORDS: Acute facial paralysis - Electrophysiological test - Electroneurography · Electromyography.

\section{Introduction}

Acute facial paralysis is an acute peripheral facial weakness of various etiologies and its diagnosis can be established without difficulty in patients with unexplained unilateral isolated facial weakness. However, bilateral facial paralysis is more difficult to be notified than unilateral involvement because bilateral facial paralysis makes symmetric weakness. The onset is sudden and symptoms typically peak within a few hours to days. The most common cause of acute onset unilateral peripheral facial weakness is Bell's palsy. Other etiologies include viral infection (herpes zoster virus, human immunodeficiency virus), Guillain-Barre syndrome, autoimmune disease, Lyme disease, Kawasaki disease, head or ear trauma, temporal bone fracture, barotrauma, acute or chronic

This is an Open Access article distributed under the terms of the Creative Commons Attribution Non-Commercial License (http://creativecommons. org/licenses/by-nc/3.0/) which permits unrestricted non-commercial use, distribution, and reproduction in any medium, provided the original work is properly cited. otitis media, cholesteatoma, sarcoidosis, Melkersson-Rosenthal syndrome, and cerebrovascular accident [1].

Most facial weakness is apparent to a clinician as well as a patient. Its clinical diagnosis is based on both static and dynamic facial analysis during physical examination, and attempts have been recently made to standardize an objective measurement of facial function, for example, digital photographic and videographic interactive computer systems [2-5]. Several systems of clinical measurement of facial nerve function have been devised, but since the mid-1980s, the HouseBrackmann grading system has been most widely accepted and endorsed by the American Academy of OtolaryngologyHead and Neck Surgery. However, in this grading system, regional descriptions of facial paralysis within each grade can overlap and lead to confusion in determining the appropriate grade. Therefore, modified House-Brackmann grading system [1] is made, focusing major functional criteria of the House-Brackmann system (absolute movement, synkinesis, eye closure, asymmetry at rest, and absolute paralysis). One of the limitations of both grading systems is the inadequacy 
to stratify the degree of degeneration or reflect the prognosis of facial paralysis.

For decades, clinicians have searched the prognostic tests of sufficient accuracy for acute facial paralysis. Since Esslen [6] introduced the use of electroneurography $(\mathrm{ENoG})$ in the early 1970s, the prognosis of facial paralysis has been predicted based mainly on various electrophysiologic tests, including the nerve excitability test, the maximal stimulation test, ENoG, and electromyography (EMG). In particular, ENoG can determine the percentage of degenerating nerve fibers in early phase of acute facial paralysis. Various studies [7-11] have shown that ENoG can be used to predict the prognosis of acute facial paralysis and May and Shambaugh [12] reported that degeneration $\leq 25 \%$ within the first 2 weeks of onset indicated a satisfactory recovery of the facial function in $98 \%$ of Bell's palsy cases.

This review article stated the clinical efficacy, advantages and disadvantages of ENoG as the prognostic test of acute facial paralysis. It also described the comparison with other electrophysiological test, including nerve excitability test (NET), maximal stimulation test (MST), and EMG.

\section{Review}

\section{Why is the electrophysiological test important?}

Facial paralysis can leave aesthetic and functional sequelae to patients and it is very important to patients as well as clinicians to choose the best treatment options and determine its prognosis. However, subjective judgment such as the House-Brackmann grading system cannot give enough or objective information on the paralysis status, especially with regard to the treatment and prognosis. Therefore, objective recording and measuring the degree of neural degeneration and resultant myopathy have been used to determine the physiologic degree of nerve injury and predict the prognosis.

Electrophysiological test is one of supportive tools for the diagnosis of neural-muscular system but not a method to diagnose the disease or confirm the etiology. Before the electrophysiological tests, the history of the facial paralysis and physical examination are preceded and considered to interpret the results of electrophysiological tests. The purposes of electrophysiological tests are to localize the lesion site along the nerve, determine the severity of the injury, and differentiate whether an injured nerve is still degenerating or regenerating.

The pathophysiology of peripheral nerve after the injury

To understand the concept of peripheral neural injury, we should understand what happens after the injury of peripher- al nerve. After the injury of peripheral nerve, pathophysiologic changes depend on the severity of the injury as well as the proximity of the injured segment to the cell body. After mild injury like neuropraxia, focal demyelination and remyelination occur. However, retrograde degeneration and regeneration of the axon occur in the case of severer injury. In mild injury, the regenerative and repair processes begin almost immediately, but nerve regeneration begins only after Wallerian degeneration has run its course in severer injury.

\section{Degeneration of the injured nerve}

Before regeneration of nerve fibers can occur, a series of degenerative processes must take place. Transection of the axon divided the nerve into proximal segment connecting to a cell body and distal one. Two segments retract in the direction opposite to the transection site each other and two axonal stumps become to be swollen because of accumulation of the cytoskeleton elements along fast axonal transport and slow axoplasmic flow. Within 24 hours post-injury, the nucleus migrates to the periphery of the cell and Nissl granules, rough endoplasmic reticulum, breaks up and disperses (chromatolysis). This chromatolysis ends in 10-21 days after the injury. Within 3 days post-injury, axonal sprout starts to grow from proximal stump and the rate of axonal regeneration is generally estimated to be $1 \mathrm{~mm}$ per day. Within 24 hours postinjury, Wallerian degeneration of axon and myelin sheath occur and Schwann cells proliferate. In Wallerian degeneration, the primary change is physical fragmentation of both axons and myelin sheath and both neurotubules and neurofilaments become disarrayed. By 48 to 96 hours post-injury, axonal continuity is lost and nerve conduction is lost. Myelin disintegration lags slightly behind that of axons but is well advanced by 36 to 48 hours. Disintegrated debris of axon and myelin sheath is removed by phagocytosis of macrophages within 12-14 days post-injury. Schwann cell plays a key role in Wallerian degeneration. It becomes active within 24 hours post-injury, divides rapidly into differentiated daughter cells, and upregulates the gene expression for proteins to assist in the degeneration and repair process. Initial role of Schwann cell is to help removal of degenerated debris of axon and myelin sheath. Schwann cell and macrophage work together for phagocytosis and clear the site of injury in a process that requires 2 week to 3 months [13-16]

\section{Regeneration of the nerve}

Regenerative and repair processes begin almost immediately, although regeneration of the severer injured nerve begins only after Wallerian degeneration has run along the nerve. For more severe nerve injuries more than $3^{\text {rd }}$ degree of Sunder- 
land classification (neurotmesis of Seddon classification), endoneurial tube is disrupted and axon cannot regenerate into its original sheath. Proliferated Schwann cells form cytoskeleton framework (Bungner band) connecting both ends of transected nerve. The earliest signs of nerve regeneration are visible changes in the cell body that mark the reversal of chromatolysis. The metabolic machinery of the cell body is reprogrammed to produce proteins and lipid needed for axonal regrowth during the regeneration process. Both fast and slow axoplasmic transports supply the cytoskeletal materials from the cell body to the sites of axonal regeneration but this process results in swelling of both stumps of transected nerve within several hours post-injury. Axonal regrowth begins as early as 24 hours post-injury. During regeneration, axonal regrowth may be impeded by fibrous tissues and regenerated nerve with myelin sheath can make scar neuroma. Multiple axon sprouts may enter into each endoneurial sheath, even in milder injuries, that do not involve destruction of the sheath itself. However, only one axon sprout becomes to be myelinated. Sometimes, axon sprout may enter into endoneurial tube other than its own. If one axon sprout enters into endoneurial sheath other than its own, we called it as "simple misdirection". If multiple branches of one axon sprout enter into endoneurial sheath other than its own, we called it as "complex misdirection". Clinical examples of complex misdirection are synkinesis and mass movement. Axon sprout which does not enter into any endoneurial sheath becomes atrophic and breaks down $[17,18]$.

\section{The pathophysiologic concept of peripheral neural injury}

To interpret the results of electrophysiological tests, we should understand the pathophysiologic concept on the degree of neural injury. In 1943, Seddon [19] described three basic types of peripheral nerve injury that include neuropraxia, axonotmesis, and neurotmesis (Fig. 1). In 1951, Sunderland [20] expanded Seddon's classification to five degrees of peripheral nerve injury (Fig. 1). The $1^{\text {st }}$ degree is essentially the same as neuropraxia of Seddon classification. The $2^{\text {nd }}$ degree is same as axonotmesis of Seddon classification. The $3^{\text {rd }}$ degree is axonotmesis as well as the disruption of endoneurium (intact epi- and perineurium). The $4^{\text {th }}$ degree is axonotmesis, as well as the disruption of endo- and perineurium (intact epineurium). The $5^{\text {th }}$ degree is same as neurotmesis of Seddon classification (complete transection). Sunderland classification is more suitable for the acute traumatic facial paralysis and Seddon classification is for acute inflammatory facial paralysis, such as Bell's palsy. Neuropraxia means local injury of myelin with the axon still intact and functional and is considered as a temporary paralysis of the nerve fiber. So, the nerve action potential can propagate along the nerve in the case of neuropraxia (Seddon classification) or 1st degree injury (Sunderland classification). Major weakness of electrophysiological tests is that they cannot differentiate between axonotmesis and neurotmesis (Seddon classification) or between $2^{\text {nd }}$ to $4^{\text {th }}$ degree injuries (Sunderland classification).

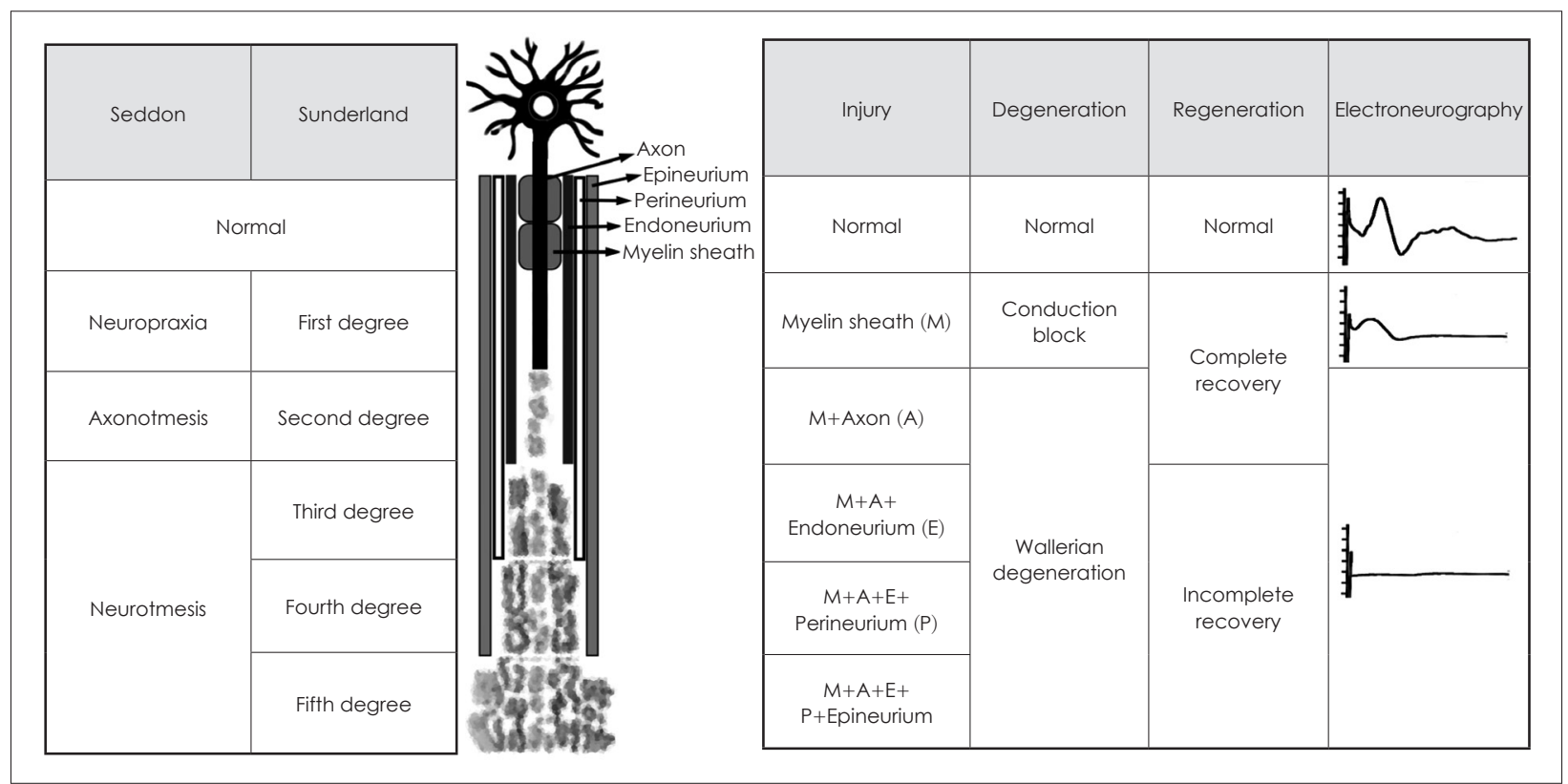

Fig. 1. Overview of Seddon and Sunderland classifications. 
Which electrophysiological tests are used to evaluate the facial nerve?

NET, MST, ENoG and EMG are representative electrophysiological tests, which have been widely used clinically. Among these, NET and MST involve the examiner's visual evaluation of electrically-elicited facial movement. Therefore, these two tests have possible subjectivity. NET is the oldest and best known electrophysiological test with well-established clinical efficacy, introduced by Laumans and Jongkees in 1963 [21]. During this test, the lowest current eliciting a facial twitch is defined as the threshold of excitation and the difference in thresholds between the two sides is calculated. During MST, facial movement of the paralyzed side is compared with that of the normal side at the level of maximal stimuli (current level at which the greatest amplitude of facial movement is seen at the normal side). Contrary to NET, MST and ENoG, EMG is the sole electrophysiological test which is very useful after loss of nerve excitability and completeness of degeneration. After 2 to 3 weeks after acute facial paralysis, tests of electrical stimulation (NET, MST, and ENoG) are no longer useful. After 10 to 14 days post-onset, fibrillation potentials or positive sharp waves seen on EMG can confirm the degeneration of facial nerve. In addition, polyphasic reinnervation potentials more useful, which may be seen as early as 4 to 6 weeks after the onset of paralysis.

\section{What is the ENoG?}

The ENoG records compound muscle action potential (CMAP) of facial muscle, which is elicited electrically. The facial nerve is stimulated transcutaneously at the stylomastoid foramen using a bipolar stimulating electrode. Responses to maximal electrical stimulation of the two sides recorded electrically by the second bipolar electrode pair placed in several sensory regions of facial nerve branches and compared between both sides. Contrary to NET or MST, ENoG calculates electrically-evoked responses objectively for the amplitude of electrically-evoked response is measured in $\mathrm{mV}$.

Typically, ENoG is delayed until 72 hours post-onset of paralysis because it takes some 72 hours for Wallerian degeneration to propagate from intratemporal portion (injured site) to portion distal to the stylomastoid foramen (electricallystimulated site during ENoG). Other end of the timing window is the 21 day post-onset of paralysis. After this time, the nerve excitability is lost and the nerve degeneration is completed. In addition, ENoG may be interfered by possible collateral nerves which are regenerated after 2 weeks post-onset. Clinicians should keep in mind that ENoG should be performed for the first time at about 72 hours post-onset and again at 3 to 5 day intervals until a trend and confirmation can be determined. It is good to test the patient serially until a plateau can be determined.

The factors influencing the test results are 1) electrical impedance between electrodes and skin, 2) conduction velocity of the facial nerve, 3) transmission velocity at the junction of neuromuscular junction, 4) propagation velocity along the facial muscle, 5) the degree of synchrony of the facial muscle fibers, and 6) the population of facial nerve fibers still intact. Other factors include sweat and oil status of the skin, diameter of the electrodes, distance between electrodes, location of the electrodes, pressure on the electrodes, skin impedance, and response of the masseter muscles [22,23]. When proper ENoG response is not elicited, clinicians should consider 1) whether or not the electrodes are detached, 2) whether or not the stimulating electrode is malfunctioning, 3) whether or not the facial nerve is totally degenerated, or 4) whether or not the trigeminal nerve is stimulated.

During analysis of ENoG results, latency is not important. Of primary importance is the amplitude of CMAP of the facial nerve. The percentage response of ENoG is defined as a percentage of the amplitude of the paralyzed side divided by the amplitude of the normal side. Alternatively, percentage degeneration is calculated by 1 minus percentage response. The test-retest variability of ENoG amplitude is 6-20\% [22,24]. ENoG test-retest variability between sides is known to be $3-20 \%$ in normal healthy subjects and asymmetry more than $30 \%$ is considered as a clinically significant difference [25].

The major advantage of ENoG is its usefulness to predict the prognosis in early stage of acute facial paralysis. Because conduction block and axonal degeneration progress together in Bell's palsy, ENoG has the advantage of evaluating the portion of the axons which are not degenerated. Esslen [26] and Fisch [27] demonstrated that in the cases with Bell's palsy of $95 \%$ degeneration, the prognosis for return of facial nerve function was greatly reduced, with a $50 \%$ chance of unfavorable recovery. May, et al. [8] reported the results of ENoG performed within the first 10 days after onset in cases with complete paralysis, which showed that percentage response of less than $10 \%$ was highly correlated with incomplete recovery, whereas percentage degeneration of less than $25 \%$ has a $98 \%$ chance of a satisfactory recovery. Fisch [28] stated that percentage degeneration of $95 \%$ within 2 weeks gave a $50 \%$ chance of a poor recovery and more gradual decrease in ENoG amplitude was related with a much better prognosis.

As mentioned above, it is important to repeat ENoG until a trend and confirmation can be determined. For example, in the case with percentage degeneration of $50 \%$ but complete palsy, we can draw that this facial paralysis may result from conduction block and the prognosis may be excellent. ENoG 
is useful to predict the prognosis only during the first 2 weeks after the onset. After then, accurate prediction using ENoG is difficult because the regeneration process already begins at the proximal end or the collateral neural circuit can develop from a healthy nerve around a degenerated nerve.

\section{Conclusion}

ENoG is considered the most valuable test to predict the prognosis and its main indication is acute complete facial paralysis. Both the percentage degeneration and rate of degeneration are prognostic indicators. In Bell's palsy, percentage degeneration of more than $90 \%$ within 14 days post-onset of complete paralysis indicates poor prognosis in more than $50 \%$ of cases.

Although electrophysiological tests provide useful neurophysiological information and serve as a guide to the management of acute facial paralysis, they have some limitations. They cannot distinguish between axonotmesis and neurotmesis and are not useful in cases of incomplete paralysis. In addition, they cannot give any useful information within first 72 hours after the onset.

\section{Conflicts of interest}

The author has no financial conflicts of interest.

\section{REFERENCES}

1) Mattox DE. Clinical disorders of the facial nerve. In: Flint PW, Haughey BH, Lund VJ, Niparko JK, Richardson MA, Robbins KT, et al., editors. Cummings Otolaryngology: Head \& Neck Surgery. 6th ed. Philadelphia, PA: Elsevier Science Health Science;2014. p.2617-28.

2) Linstrom CJ. Objective facial motion analysis in patients with facial nerve dysfunction. Laryngoscope 2002;112(7 Pt 1):1129-47.

3) Neely JG, Wang KX, Shapland CA, Sehizadeh A, Wang A. Computerized objective measurement of facial motion: normal variation and test-retest reliability. Otol Neurotol 2010;31:1488-92.

4) Frey M, Tzou CH, Michaelidou M, Pona I, Hold A, Placheta E, et al. 3D video analysis of facial movements. Facial Plast Surg Clin North Am 2011;19:639-46, viii.

5) Hadlock TA, Urban LS. Toward a universal, automated facial measurement tool in facial reanimation. Arch Facial Plast Surg 2012;14: 277-82.

6) Esslen E. Electrodiagnosis of facial palsy. In: Miehlke A, editor. Surgery of the Facial Nerve. Philadelphia, PA: W.B. Saunders;1973. p.45-51.

7) Thomander L, Stälberg E. Electroneurography in the prognostication of Bell's palsy. Acta Otolaryngol 1981;92:221-37.

8) May M, Blumenthal F, Klein SR. Acute Bell's palsy: prognostic value of evoked electromyography, maximal stimulation, and other electrical tests. Am J Otol 1983;5:1-7.

9) Fisch U. Prognostic value of electrical tests in acute facial paralysis. Am J Otol 1984;5:494-8.

10) Sillman JS, Niparko JK, Lee SS, Kileny PR. Prognostic value of evoked and standard electromyography in acute facial paralysis. Otolaryngol Head Neck Surg 1992;107:377-81.

11) Smith IM, Maynard C, Mountain RE, Barr-Hamilton R, Armstrong M, Murray JA. The prognostic value of facial electroneurography in Bell's palsy. Clin Otolaryngol Allied Sci 1994;19:201-3.

12) May M, Shambaugh GE. Facial nerve paralysis. In: Paparella MM, Shumrick DA, Gluckman JL, Meyerhoff W, editors. Otolaryngology. 3rd ed. Philadelphia, PA: W.B. Saunders;1991. p.1106.

13) Sunderland S, Bradley KC. Endoneurial tube shrinkage in the distal segment of a severed nerve. J Comp Neurol 1950;93:411-20.

14) Kreutzberg GW, Schubert P. Volume changes in the axon during regeneration. Acta Neuropathol 1971;17:220-6.

15) May M. Anatomy of the facial nerve (spatial orientation of fibers in the temporal bone). Laryngoscope 1973;83:1311-29.

16) Sunderland S. The anatomy and physiology of nerve injury. Muscle Nerve 1990;13:771-84.

17) Campbell WW. Evaluation and management of peripheral nerve injury. Clin Neurophysiol 2008;119:1951-65.

18) Fisch U, Felix H. On the pathogenesis of Bell's palsy. Acta Otolaryngol 1983;95:532-8.

19) Seddon HJ. Three types of nerve injury. Brain 1943;66:238-87.

20) Sunderland S. A classification of peripheral nerve injuries producing loss of function. Brain 1951;74:491-516.

21) Laumans EP, Jongkees LB. On the prognpsis of peeripheral facial paralysis of endotemporal origin. Ann Otol Rhinol Laryngol 1963; 72:621-36.

22) Hughes GB, Nodar RH, Williams GW. Analysis of test-retest variability in facial electroneurography. Otolaryngol Head Neck Surg 1983;91:290-3.

23) Gantz BJ, Gmuer AA, Holliday M, Fisch U. Electroneurographic evaluation of the facial nerve. Method and technical problems. Ann Otol Rhinol Laryngol 1984;93(4 Pt 1):394-8.

24) Coker NJ. Facial electroneurography: analysis of techniques and correlation with degenerating motoneurons. Laryngoscope 1992;102: 747-59.

25) Raslan WF, Wiet R, Zealear DL. A statistical study of ENoG test error. Laryngoscope 1988;98(8 Pt 1):891-3.

26) Esslen E. The acute facial palsies: investigations on the localization and pathogenesis of meato-labyrinthine facial palsies. Berlin: Springer-Verlag;1976.

27) Fisch U. Maximal nerve excitability testing vs electroneuronography. Arch Otolaryngol 1980;106:352-7.

28) Fisch U. Surgery for Bell's palsy. Arch Otolaryngol 1981;107:1-11. 